\title{
Productividad de recursos humanos, innovación de producto y desempeño exportador: Una investigación empírica
}

\author{
Rafael Reina Valle \\ Universidad de Sevilla \& Universidad Europea de Madrid (Spain) \\ rreina@us.es
}

Received December, 2015

Accepted March, 2016

\section{Resumen}

Objeto: El objetivo de esta investigación es examinar las relaciones entre la productividad de los recursos humanos (RRHH), la innovación de producto y el desempeño exportador de la PYME manufacturera española. La proposición central de esta investigación es, que la productividad de los RRHH facilita el desarrollo de innovaciones de producto, para incrementar el desempeño exportador de las empresas.

Diseño/metodología/enfoque: El modelo conceptual propuesto, se contrasta empleando la técnica de la modelización de ecuaciones estructurales, basada en la varianza y el método de estimación de los mínimos cuadrados parciales, sobre una muestra de 858 PYMES manufactureras españolas.

Aportaciones y resultados: El análisis empírico da apoyo a nuestra teoría.

Limitaciones: Esta investigación sólo se centra en el rol de la innovación de producto, en la relación entre la productividad de los RRHH y el desempeño exportador. También sería interesante estudiar el papel mediador de capacidades organizativas, basadas en otros tipos de innovaciones (p.e., la innovación en procesos, organizativa o en marketing).

Implicaciones prácticas: Sugerir a los empresarios de PYMES, que incrementos en la productividad de los RRHH puede llevar a que dichos impactos sean capturados por la 
innovación, dando lugar a la generación de nuevos productos, que conllevará shocks de demanda, y en consecuencia de un mayor incremento de la exportación de sus empresas.

Originalidad / Valor añadido: Esta investigación explica conceptualmente y demuestra empíricamente, que la innovación de producto es un mecanismo intermedio clave, a través del cual, la PYME manufacturera española, puede incrementar sus resultados de exportación, explotando la productividad de su base de talento productiva.

Palabras clave: Productividad de los recursos humanos, Innovación de producto, Desempeño exportador, PLS, PYMES manufactureras

Códigos JEL: F1, L21, O32

Title: Human resource productivity, product innovation, and export performance: An empirical investigation

\section{Abstract}

Purpose: The objective of this research is to examine the relationships between human resource (HR) productivity, product innovation, and export performance of the Spanish manufacturing SMEs. Our central proposition is that HR productivity enables the development of product innovation to increase firms' export performance.

Design/methodology: The proposed conceptual model, is tested using the technique of variance-based structural equation modeling and the estimation method of partial least squares, on a sample of 858 Spanish manufacturing SMEs.

Findings: The empirical analysis supports our theory.

Research limitations/implications: This research only focuses on the role of product, innovation in the relationship between HR productivity and export performance. Further research may study the mediating role of organizational capabilities based on other types of innovation (e.g., process, organizational, or marketing innovation).

Practical implications: Suggest to entrepreneurs of SMEs, that increased HR productivity can lead to such impacts are captured by innovation, resulting in the generation of new products, which will lead demand shocks, and consequently a higher increase in firms' exports. 
Originality/value: This research explains conceptually and empirically shows, that product innovation is a key intermediate mechanism, through which the Spanish manufacturing SME can increase its export results by exploiting the productivity of its talent base.

Keywords: Human resource productivity, product innovation, export performance, PLS, manufacturing SMEs

Jel Codes: F1, L21, O32

\section{Introducción}

Durante los años de la crisis ha crecido la exportación con fuerza, a su vez también el número de empresas exportadoras. La exportación de productos es una estrategia de supervivencia empresarial, cuando se agotan las ventas en el mercado nacional. Pero la mayor parte de estas nuevas empresas exportadoras, exportan de manera esporádica, no regular. Entre 2008 y 2013 el número de empresas exportadoras en España, aumentó en cerca de 50,000 (ICEX, 2014), pero el número de empresas exportadoras regulares aumentó en sólo 1,522.

En apenas tres décadas, la economía española ha pasado de ser una economía altamente protegida, a ser abierta, con un número muy elevado de empresas operando fuera en las distintas formas de internacionalización existentes. En la actualidad, nuestro país presenta una tasa de apertura al exterior, similar a los países de nuestro entorno, como Francia, Alemania, Italia o el Reino Unido. Pero debemos tener presente que en España, sólo el 29\% de las PYMES sobreviven al quinto año de vida (OCDE, 2015), y que el $80 \%$ de las nuevas empresas mueren en su primer año de vida (Andrés, 2011). Nos encontramos ante un doble fenómeno, por un lado, todavía hay empresas que siguen percibien do la venta al exterior como una oportunidad puntual, y por otro, de todas las empresas que inician su andadura exportadora, muchas abandonan, debido a que les falta continuidad en su actividad exportadora. En este sentido, estudiar qué variables afectan al desarrollo de actividades exportadoras y a su éxito, es un tema de investigación con impacto en el mundo real y académico.

La literatura previa se ha centrado, en el estudio sectorial de las características de la empresa exportadora y en otros determinantes del desempeño exportador (p.e., Martín \& Rodríguez, 2009). Sin 
embargo, el estudio del rol de la innovación en las actividades exportadoras de la empresa, es extraordinariamente limitada. Este gap es el que pretende completar esta investigación.

El objetivo de este trabajo es examinar las relaciones entre la productividad de los recursos humanos (RRHH), la innovación de producto y el desempeño exportador de la PYME manufacturera española.

Usando la teoría basada en las capacidades organizativas (Barney, 1991; Teece \& Pisano, 1994; Teece, Pisano \& Schuen, 1997; Teece, 2007), la proposición central de esta investigación es, que la productividad de los RRHH facilita el desarrollo de innovaciones de producto, para incrementar el desempeño exportador de las empresas. El modelo conceptual propuesto, se contrasta empleando la técnica de la modelización de ecuaciones estructurales, basada en la varianza y el método de estimación de los mínimos cuadrados parciales, sobre una muestra de 858 PYMES manufactureras españolas. El análisis empírico sugiere, que la productividad de los RRHH facilita el desarrollo de innovaciones de producto, para incrementar el desempeño exportador. En este sentido, la innovación de producto es un mecanismo intermedio clave a través del cual, la mayor productividad de los RRHH, se convierte en un mayor desempeño exportador de la PYME manufacturera española.

\section{Modelo conceptual propuesto y desarrollo de hipótesis}

\subsection{La teoría de las capacidades organizativas}

La teoría de las capacidades organizativas sugiere, que la varianza de la competitividad de las empresas, viene explicada por las distintas capacidades que tienen las empresas. Esta teoría constituye una extensión de la teoría basada en los recursos (p.e., Barney, 1991) que enfatiza, que la clave no es la posesión de los recursos organizativos, sino cómo y para qué se usan los mismos en la empresa (p.e., Prahalad \& Hamel, 1990).

La competitividad generada en las últimas décadas, ha forzado a las organizaciones a renovar, adaptar y reconfigurar sus recursos al ritmo del mercado. Así, surge la teoría de las capacidades dinámicas (p.e., Teece et al., 1997; Eisenhardt \& Martin, 2000). Las capacidades dinámicas se refieren a la habilidad de la empresa de diseñar, integrar y reconfigurar su base de recursos como respuesta a los cambios en su entorno empresarial (p.e., Benítez, Lloréns \& Férnandez, 2015). Algunos ejemplos de capacidades dinámicas, son la capacidad de desarrollo de nuevos productos y la capacidad de realizar alianzas estratégicas (p.e., Eisenhardt \& Martin, 2000). Otro tipo de capacidades organizativas, son las 
capacidades operativas/funcionales, que se refieren a la habilidad de la empresa, de ejecutar rutinas para resolver problemas operativos e implementar su estrategia de operaciones (p.e., Benítez \& Ray, 2012). La teoría de las capacidades organizativas, proporciona un marco teórico/conceptual útil para la conceptualización de la productividad de los RRHH y la innovación de producto, así como para proporcionar los argumentos que vinculan, innovación de producto y el desempeño exportador de la PYME (p.e., Teece et al., 1997; Núñez, Grande \& Lorenzo, 2015).

\subsection{Proposición central de la investigación}

El modelo conceptual de este trabajo de investigación propone, que la productividad de los RRH facilita el desarrollo de innovaciones de producto, para incrementar el desempeño exportador. En este sentido, la innovación de producto puede ser un mecanismo intermedio a través del cual, la mayor productividad de los RRHH puede explotarse, para desarrollar más y mejores productos innovadores, incrementando el desempeño exportador de la PYME manufacturera española.

El modelo conceptual de estudio se muestra en la Figura 1.

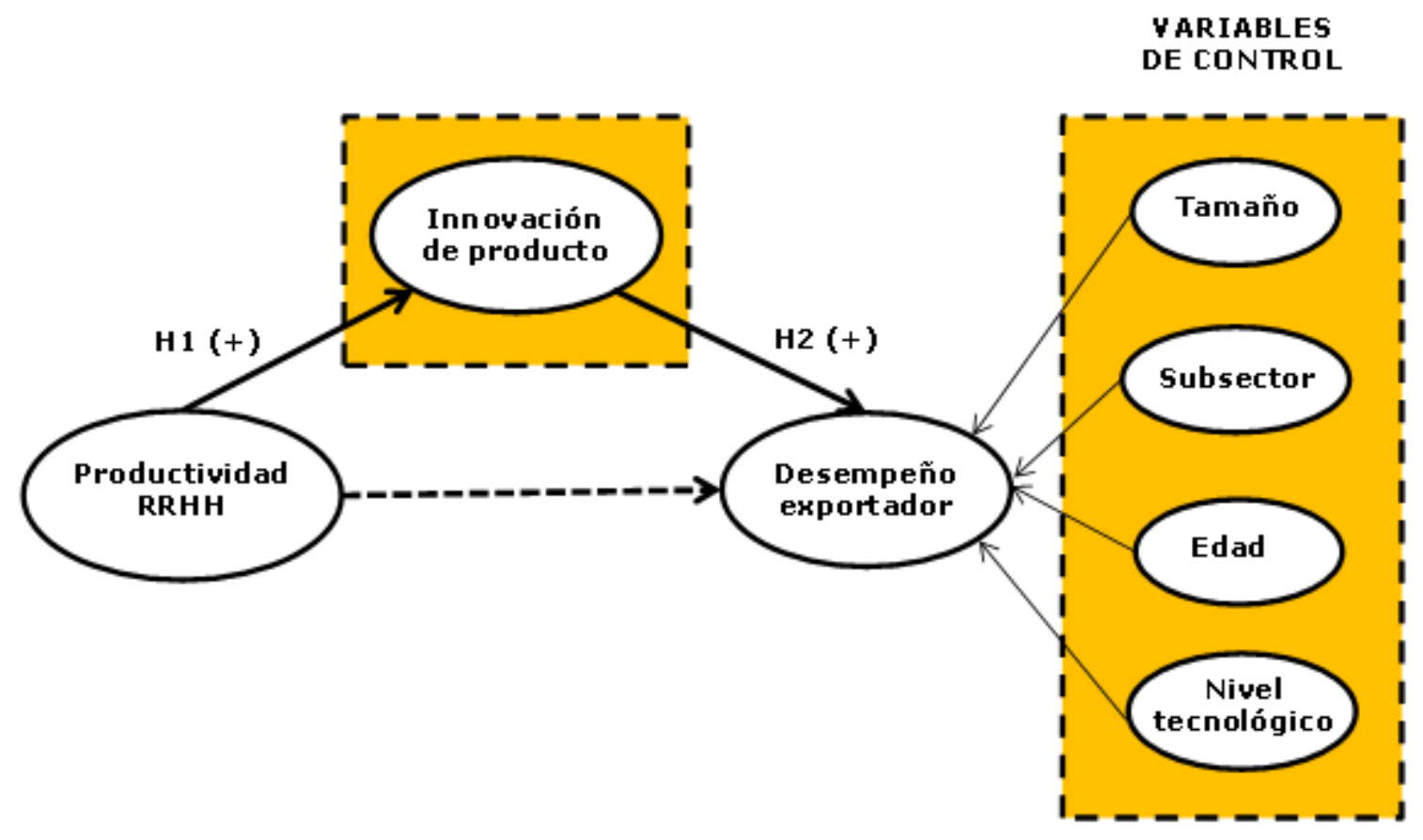

Figura 1. Modelo de investigación 


\subsection{Productividad de los RRHH e innovación de producto}

El factor humano está presente en todos los momentos del hecho productivo, ya que se requiere de la participación de las personas y de una permanente relación social laboral, para obtener resultados. Al respecto, numerosos autores han planteado, la relación e influencia que ejerce el factor humano en la productividad y el desempeño organizativo (Kemppilä \& Lönnqvist, 2003; Saari \& Judge, 2004). La formación y el entrenamiento, elevan o están relacionados directamente con la productividad; el capital humano es crucial para el éxito de las organizaciones y se convierte en una ventaja competitiva sostenible, dando como resultado el mejor retorno de su inversión (Lagarda \& Urquidy, 2007; Luthans \& Youssef, 2004). En nuestro trabajo, entenderemos la productividad de los RRHH, como un indicador de la eficacia organizativa y de la generación de productos, es decir, como aquella capacidad del capital humano que incorpora la experiencia, habilidades y los conocimientos adquiridos, para encontrar la mejor forma de hacer a la empresa más eficiente y productiva (Quijano, 2006), de tal forma que a medida que los trabajadores están más formados, adquieren más habilidades, realizando las tareas de manera más eficiente, impactando directamente en la capacidad innovadora de la firma.

La innovación de producto, se refiere a la introducción de un producto (bien o servicio) nuevo o significativamente mejorado, en cuanto a sus características básicas o el uso al cual se destina, ya sea para la propia empresa, como para el mercado en el que opera. Las innovaciones de producto, incluyen las mejoras significativas de las especificaciones técnicas, de los componentes y de los materiales, de los programas informáticos integrados, de la facilidad de uso u otras características funcionales (OCDE, 2005). En cambio, las innovaciones de proceso, son cambios significativos en los métodos de producción y de distribución.

Algunos autores, apuntan a un incremento del valor añadido por trabajador, a medida que aumentan el número de mercados externos abordados (Eaton Kortum \& Kramarz, 2011). Otros van más allá, e indican que a través de mejoras en la productividad, se consigue incrementar también la competitividad de las empresas (Visintin, Di Meglio, Rubalcaba \& Cuadrado, 2008). La idea es que se produce una autoselección de empresas, pues sólo sobreviven a la entrada aquellas que consiguen tener mejor productividad, y compensan el coste hundido de la inversión de penetración en el mercado extranjero. Es decir, sólo aquellas empresas que son lo bastante productivas como para soportar los costes de entrada y la intensa competencia, van a poder entrar en los mercados exteriores vía exportación. 
Dicho lo anterior, proponemos que la productividad de los RRHH puede ayudar a la empresa a mejorar sus actividades de innovación, y a realizar innovaciones de producto. Por lo tanto:

Hipótesis 1 (H1): Existe una relación positiva y significativa, entre la productividad de los recursos humanos de la PYME manufacturera y la innovación de producto.

\subsection{Innovación de producto y desempeño exportador}

Nos encontramos ante una economía globalizada, y ante una demanda del mercado interior en plena crisis, es imprescindible para las empresas, ser más competitivas en los mercados exteriores, si pretenden sobrevivir a esta situación.

Rialp y Rialp (2001), sugieren que existen tres aspectos clave en cualquier proceso de internacionalización empresarial:

- la selección del objeto de la internacionalización (productos y/o recursos);

- el ámbito geográfico (país, mercados y/o nichos) y

- el mecanismo de entrada (mediante exportación indirecta o directa, a través de acuerdos contractuales como licencias y/o franquicias, o por medio de inversiones directas en el exterior como filiales y/o empresas conjuntas).

Desde esta óptica, la exportación ha sido el modo más popular y primario de internacionalización, utilizado principalmente por las PYMES, debido a su menor tamaño relativo, dado que se requiere una menor cantidad de recursos (financieros, humanos, etc.), involucrando un riesgo menor (Leonidou, Katsikeas \& Samiee, 2002). En este sentido, el producto es uno de los objetos de la internalización. Por otro lado, la exportación es la forma más habitual de internacionalización de la PYME, por lo que el estudio del impacto de la innovación de producto sobre el desempeño exportador, es racional y clave.

Numerosos estudios respaldan la relación entre el desempeño exportador de las empresas y su nivel de competitividad, contribuyendo a unos mejores resultados empresariales. Las evidencias obtenidas muestran, por un lado, que los resultados empresariales mejoran como consecuencia del inicio de exportaciones (Katsikeas, Leonidou \& Morgan, 2000), y por otro, que unos buenos resultados previos, facilitan tanto el inicio de exportaciones, como una mayor presencia en el exterior de las firmas (García \& Avella, 2008). En este sentido, consideramos el desempeño exportador de la PYME, como el 
compromiso de la dirección de la firma en su actividad internacional (p.e., Czinkota \& Johnston, 1983; Núñez et al., 2015).

Una empresa innovadora que es capaz de desarrollar un nuevo producto, se puede permitir aumentar su demanda y por tanto incrementar su margen de beneficio, colocando a la firma innovadora, en una posición ventajosa en los mercados foráneos. Así mismo, las empresas también pueden aumentar la demanda, mediante la diferenciación de sus productos, abriendo nuevos mercados e influyendo sobre la demanda de productos existentes. Las empresas con mayores niveles de innovación de producto, suelen estar sometidas a presiones competitivas más fuertes, lo que les redundará en mayores porcentajes de ventas en el extranjero.

La innovación de producto puede por tanto incrementar el desempeño exportador de la empresa. $\mathrm{Al}$ mismo tiempo, la $\mathrm{I}+\mathrm{D}+\mathrm{i}$ parece desempeñar un papel relevante, en la explicación de las decisiones sobre la exportación y el volumen de exportaciones a realizar, existiendo muchos estudios que asocian innovación y exportación (Basile, 2001; Bernard \& Jensen, 2004). Por ejemplo, Kugler y Verhoogen (2008) muestran, que los productos de las empresas exportadoras tienen precios más altos, lo que sugiere, que los productos más novedosos y de mayor calidad son los que se exportan.

Damanpour y Aravind (2006), argumentan que la habilidad de las empresas para generar ventajas competitivas, así como para conseguir un desempeño superior, depende en gran medida de su capacidad para la innovación en productos. En esa misma dirección, un conjunto de trabajos que estudian la inserción internacional de firmas de países latinoamericanos (Sánchez, Hernández \& Prada, 2004; Molina-Domene \& Pietrobelli, 2012), plantean que el éxito exportador se logra a lo largo de un sendero evolutivo, que requiere tiempo, y se sustenta en el desarrollo de importantes capacidades tecnológicas y productivas. Boscherini y Yoguel (1996), exploran la relación entre capacidades innovativas y desempeño exportador en PYMES industriales argentinas. La capacidad innovativa incluye un conjunto de dimensiones tales como las actividades de capacitación, la gestión de la calidad, la interacción con otros agentes y la calificación del personal. Otros autores encuentran, que las capacidades tecnológicas constituyen un elemento discriminador significativo para la actividad exportadora de las firmas (Moori, Milesi, Robert \& Yoguel, 2007; Molina-Domene \& Pietrobelli, 2012). Por otro lado, un conjunto de artículos exploran la relación entre innovación y exportaciones, a partir de los resultados (producto y proceso). Becker y Egger (2013) encuentran, que tanto las innovaciones de producto como de proceso, tienen un efecto positivo sobre la propensión a exportar. 
Lo anterior sugiere, una relación positiva entre la innovación de producto y el desempeño exportador. Por lo tanto:

Hipótesis 2 (H2): Existe una relación positiva y significativa, entre la innovación de producto y el desempeño exportador.

\section{Metodología}

\subsection{Datos y medidas}

Desde el año 2003 se dispone en España de un Panel de Innovación Tecnológica (PITEC), se trata de una base de datos de tipo panel, que permite el seguimiento de las actividades de innovación tecnológica de las empresas españolas, resultado del esfuerzo conjunto del Instituto Nacional de Estadística (INE) y la Fundación Española para la Ciencia y la Tecnología (FECYT), junto con el asesoramiento de un grupo de expertos académicos. PITEC está formado por datos provenientes de observaciones de empresas. Su realización es de carácter anual, permitiendo el análisis estático de la innovación en el país, en un momento concreto, o el análisis dinámico, comparando dos periodos de tiempo, para comprobar tendencias, evoluciones, etc. Los resultados del cuestionario son revisados anualmente, pudiéndose utilizar para analizar el comportamiento del tejido empresarial en materia de innovación.

La base de datos generada, se encuentra a disposición de los investigadores en el portal de FECYT, en la dirección http://icono.fecyt.es/PITEC/Paginas/por que.aspx. Existe un fichero de datos para cada año t, y para cada formato de datos. El número de filas (o registros) del fichero del año t, es igual al número de empresas que PITEC ha abarcado en total hasta este momento. Existe una larga lista de investigadores que han utilizado el panel PITEC en sus estudios (p.e., Jaumandreu, 2009).

Tomamos como universo, la población de empresas españolas procedentes de la base de datos PITEC, del año 2011 y 2013, que consta en su totalidad de 12,839 empresas. Esta investigación se centra en las PYMES manufactureras españolas, que exportan con regularidad por dos razones:

- las PYMES son clave para el tejido empresarial español;

- la actividad exportadora de las PYMES españolas es crítica para su supervivencia, dado el agotamiento que ha sufrido la venta de sus productos en el mercado nacional. 
Para ello, se seleccionaron aquellas empresas manufactureras que eran PYMES, es decir, que tenían menos de 250 empleados (Unión Europea, 2003). En la selección de la muestra de estudio se han considerado tres criterios. El primer criterio ha sido la regularidad de las empresas en el comercio exterior, el segundo ha sido el volumen de este comercio exterior en relación a su cifra total de ventas, y el tercer criterio está relacionado con su carácter innovador. Atendiendo al primer aspecto, se han considerado las PYMES con una experiencia exportadora regular, es decir, con al menos cinco años consecutivos a contar desde el año 2009 hasta el año 2013. En relación al segundo aspecto, se ha considerado las PYMES con una cifra de negocio exterior, superior o igual al 50\% de su cifra total de negocio. El tercer y último aspecto tenido en cuenta, ha sido su compromiso innovador, para ello, se han considerado también las PYMES, que han desarrollado al menos una innovación de cualquiera de los siguientes cuatro tipos de innovación: innovación de producto, innovación de proceso, innovación organizativa e innovación de marketing (p.e., OCDE, 2005). Una vez eliminados los casos con incidencias (empresas de alta temporalidad sectorial, cambio de actividad o abandono de la misma, empresa en fase de regulación de empleo o en fase de liquidación, etc.), la muestra final objeto de estudio, quedó formada por 858 PYMES manufactureras exportadoras regulares (cinco años consecutivos), intensivas en exportación e innovadoras.

\subsection{Medición de las variables}

Los investigadores utilizan múltiples indicadores en sus constructos, ya que esta aproximación representa el mejor camino para asegurar la validez y la fiabilidad (Cook, Campbell \& Day, 1979). Paralelamente, se ha de considerar lo que Fornell (1982) denomina relaciones epistemológicas, es decir, el vínculo entre los constructos y sus indicadores. Básicamente se distinguen dos tipos de vinculación. Por un lado, la que señala que los indicadores, son un reflejo del constructo teórico no observado al que se encuentran ligados, de tal forma, que el constructo da lugar a aquello que observa. Por otro, la que determina que los indicadores o medidas, causan o dan lugar al constructo. En el primer caso estaríamos hablando de indicadores reflectivos, mientras que en el segundo caso nos encontramos con los denominados indicadores formativos, los cuales no tienen por qué correlacionarse (co-variar) entre sí, aunque pueden hacerlo, además los indicadores formativos, no son intercambiables entre sí, y eliminar algún indicador altera el significado teórico del constructo (Jarvis, Mackenzie \& Podsakoff, 2003). 
Todos los constructos utilizados en este trabajo han sido especificados como formativos (p.e., Benítez \& Ray, 2012). Todos los datos y medidas proceden de la base de datos PITEC. A continuación se detallan los constructos incluidos en el modelo propuesto en esta investigación.

- Variables independientes:

- Productividad de los RRHH. Este constructo ha sido medido mediante una única variable observada, y viene dada por la cifra de negocios total de la PYME durante el año 2011, dividida por el número de trabajadores durante ese año.

- Innovación de producto. Este constructo ha sido medido mediante dos variables observadas, una de ellas mide el término innovación en relación con el desarrollo de nuevos productos, desde una óptica interna, es decir, de las novedades o cambios que introduce la empresa en sus productos (Munuera \& Rodríguez, 2007), y la segunda variable observada, mide la innovación de productos desde una perspectiva externa o de mercado, esto es, como la introducción de productos que suponen alguna novedad para el mercado (Garcia \& Calantone, 2002). Ambas medidas han sido consideradas, como porcentaje de la cifra total de negocio de la PYME para el año 2013.

- Variable dependiente:

- Desempeño exportador (medida en el año 2013). Este constructo es determinado por dos variables observadas: La intensidad exportadora, medida por el porcentaje de ventas en el mercado foráneo, en relación a la cifra total de negocio de la PYME, y por la experiencia exportadora, medida por el número de años que la empresa ha exportado desde su creación. La consideración de la intensidad exportadora, ha sido utilizada en numerosas investigaciones internacionales (p.e., Eusebio, Llonch \& Pilar, 2006). De hecho, el metaanálisis realizado por Katsikeas et al. (2000) indica que aproximadamente el 61\% de los estudios revisados, habían empleado este criterio para medir el desempeño exportador. La experiencia exportadora es considerada, como uno de los principales recursos y capacidades de las empresas en proceso de internacionalización, erigiéndose como una posible fuente de ventajas competitivas sostenibles (Baldauf, Cravens \& Wagner, 2000; Chen, Paulraj \& Lado, 2004). A través de la experiencia exportadora, las empresas incrementan gradualmente su implicación y compromiso con los mercados exteriores. Una mayor experiencia exportadora, aumenta la confianza de la empresa en los mercados internacionales y ayuda a reducir los costes, lo que favorece una mayor expansión geográfica (p.e., Leonidou, 2004; 
Chen et al., 2004). El nivel de intensidad/ventas y experiencia exportadora son dos buenos ingredientes, que determinan el desempeño exportador, por lo que su uso como proxy del desempeño exportador parece racional y adecuado en esta investigación (p.e., Zhao, Lynch \& Chen, 2010).

El efecto de la productividad de los RRHH sobre la innovación de producto y la actividad exportadora, podría necesitar de tiempo para su realización (Benítez \& Walczuch, 2012). Por esta razón, la productividad de los RRHH está medida en 2011, y la innovación de producto y el desempeño exportador en 2013. Esto garantiza el control del efecto tiempo, sobre las relaciones incluidas en el modelo propuesto en esta investigación.

\subsection{Variables de control}

Se incluyeron cuatro variables de control en el modelo conceptual propuesto. Por una parte, se han introducido tres variables de tipo estructural (tamaño organizativo, edad organizativa y subsector al que pertenece la PYME), y una variable de tipo tecnológico (nivel tecnológico según clasificación CNAE2009). El tamaño organizativo, fue medido a través del número de empleados (Braojos, Benítez \& Lloréns, 2015a; Wang, Chen \& Benitez-Amado, 2015). Las PYMES cuentan con estructuras productivas más flexibles y diseños organizativos menos burocráticos (Damanpour, 1991) y ello, les puede beneficiar a la hora de adaptarse más rápidamente a los cambios del entorno. Por su parte, las PYMES de mayor tamaño, pueden beneficiarse de economías de escala o alcance en sus actividades innovadoras (Henderson \& Cockburn, 1994). La edad de la empresa, fue medida como el número de años de actividad de la empresa. Esta variable identifica la experiencia de la empresa, siendo una proxy del conocimiento del mercado. Cuanto mayor sea la experiencia, menores serán los costes debido a las economías de aprendizaje, esto provocará una mayor estimulación de cara a aumentar la importancia de sus operaciones en el exterior. El subsector se midió a partir de una variable que proporciona PITEC, que identifica la rama o subsector de actividad de la PYME. Finalmente, hemos decidido introducir el nivel tecnológico de la firma, según la clasificación sectorial definida por CNAE-2009. Esta variable se encuentra muy relacionada con la capacidad de innovación tecnológica de la empresa, y explica en gran medida el potencial innovador de la misma. En el Anexo A, se pueden consultar los nombres del constructo, la definición de la medida y la fuente del dato. 


\subsection{Análisis empírico}

El modelo conceptual propuesto, es contrastado empleando el método de estimación de los mínimos cuadrados parciales (PLS), utilizando el paquete estadístico SmartPLS 2.0.M3 (Chin, Marcolin \& Newsted, 2003; Ringle, Wende \& Will, 2005). Este método de estimación es apropiado para esta investigación ya que en ella se usan constructos formativos. Dicho método no exige que los datos sigan ninguna distribución en concreto (p.e., Braojos et al., 2015a; Ruizalba, Vallespín, Martín \& Rodríguez, 2015). El análisis se llevará a cabo en dos fases: evaluación del modelo de medida y del modelo estructural.

El modelo estructural abarca las relaciones incluidas como hipótesis en el modelo propuesto (H1 y H2), así como un link entre la productividad de los RRHH y el desempeño exportador (efecto directo entre estas dos variables). El efecto total de la productividad de los RRHH sobre el desempeño exportador, viene determinado por la suma de su efecto directo (Productividad de los RRHH $\rightarrow$ Desempeño exportador), más su efecto indirecto (a través de la innovación de producto). En este sentido, realizaremos un análisis de efectos indirectos, que sirve para determinar su valor/cuantía y si el efecto indirecto es o no estadísticamente significativo (Zhao et al., 2010).

Dicha relación/análisis se muestra en la ecuación 1:

$$
\text { efecto total }=\text { efecto directo }+ \text { efecto indirecto }
$$

\section{Resultados}

\subsection{Evaluación del modelo de medida}

Los indicadores formativos no se pueden evaluar de la misma forma que los indicadores reflectivos (p.e. Benítez \& Ray, 2012). Siguiendo a Cenfetelli y Bassellier (2009), se procedió a realizar los respectivos análisis de los modelos de medida, valorando la posible multicolinealidad de los indicadores a través del Factor de Inflación de la Varianza (FIV > 5) (Hair, Ringle \& Sarstedt, 2013), así como la interpretación y significación de los pesos y de sus cargas factoriales. Si el peso es significativo y la carga también, el indicador se mantiene, y si el peso no es significativo pero su carga si lo es, también se pueden mantener los indicadores formativos (Cenfetelli \& Bassellier, 2009). 
Para determinar el nivel de significación de los pesos, cargas y coeficientes se ha usado el algoritmo del bootstrapping con 5000 sub-muestras (Braojos, Benítez \& Lloréns, 2015b) * p < .05; ** p <.01; ***p < .001 ; n.s.: No significativo.

A continuación en la Tabla 1, podemos apreciar las propiedades del modelo de medida.

\begin{tabular}{|l|l|r|r|r|}
\hline Nombre del constructo & \multicolumn{1}{|c|}{ Nombre del indicador } & \multicolumn{1}{|c|}{ FIV } & \multicolumn{1}{|c|}{ Pesos } & \multicolumn{1}{|c|}{ Carga } \\
\hline \multirow{2}{*}{ Innovación de producto } & Nuevos para la PYME & 1.000 & $0.815^{* *}$ & $0.815^{* *}$ \\
\cline { 2 - 5 } & Nuevos para el mercado & 1.000 & $0.580^{*}$ & $0.580^{*}$ \\
\hline \multirow{2}{*}{ Desempeño exportador } & Experiencia exportadora & 1.006 & $0.057^{*}$ & $0.131^{* *}$ \\
\cline { 2 - 5 } & Intensidad exportadora & 1.006 & $0.994^{* * *}$ & $0.998^{* * *}$ \\
\hline
\end{tabular}

Tabla 1. Evaluación del modelo de medida

\subsection{Evaluación del modelo estructural: Test de hipótesis}

A continuación en la Figura 2, podemos apreciar la significación estadística del modelo estructural.

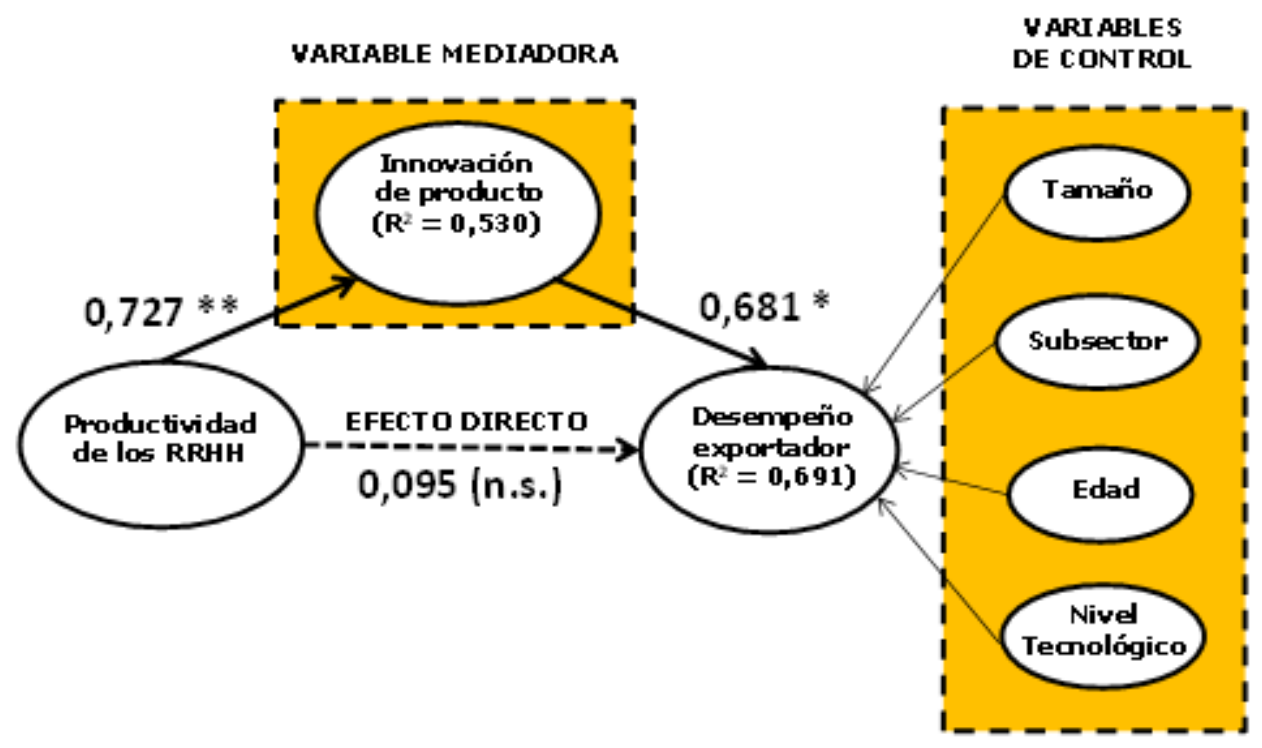

$* \mathbf{p}<.05 ; * * \mathbf{p}<.01 ; * * * \mathbf{p}<.001 ; \mathbf{n} s .2$ No significativo.

T-Student de una cola con $n-1$ grados de libertad, donde $n$ es el número de submuestras. Para $\mathbf{n}=\mathbf{5 0 0 0}$ submuestras.

Figura 2. Evaluación del modelo estructural 
En la Tabla 2, se ofrecen los resultados del modelo estructural con su correspondiente contraste de hipótesis.

\begin{tabular}{|l|c|r|r|c|}
\hline \multicolumn{4}{|c|}{ Varianza explicada por el modelo para Desempeño exportador $\mathbf{R}^{2}=0.691$} \\
\hline \multicolumn{1}{|c|}{ Hipótesis } & Signo & Coef. Path $(\boldsymbol{\beta})$ & $\begin{array}{c}\text { T-Valor } \\
\text { (Bootstrap) }\end{array}$ & Contraste \\
\hline $\begin{array}{l}\text { H1:Productividad RRHH à Innovación producto } \\
\text { H2:Innovación producto à Desempeño } \\
\text { exportador }\end{array}$ & $(+)$ & $0.727 * *$ & 2.985 & SI \\
\hline
\end{tabular}

${ }^{*} \mathrm{p}<.05 ;{ }^{* *} \mathrm{p}<.01 ;{ }^{* * *} \mathrm{p}<.001 ;$ n.s.: No significativo.

Tabla 2. Test de hipótesis de investigación

El análisis empírico da apoyo a las dos hipótesis del modelo ( $\mathrm{H} 1$ y H2) con un nivel de significación de 0.05. Así, el 53\% de la varianza de la innovación de producto, viene explicada por la productividad de los RRHH, y el 69.1\% de la varianza del desempeño exportador, viene explicado por la innovación de producto. En resumen, el análisis empírico da apoyo a nuestra teoría.

Siguiendo a Zhao et al. (2010), y a Preacher y Hayes (2008), analizamos el efecto indirecto de la mediación de la variable innovación de producto, en el impacto directo de la productividad de los RRHH, en el desempeño exportador. El efecto indirecto de la innovación de producto, en la relación entre la productividad de los RRHH sobre el desempeño exportador, es de 0.497 , con un intervalo de confianza de 0.007 - 0.839, es decir, significativo al 95\% (Preacher \& Hayes, 2008). El efecto total es de 0.592 (efecto directo + efecto indirecto $=0.095+0.497$ ). Como el efecto directo de la productividad de los RRHH sobre el desempeño exportador, no es significativo (0.095), mientras que el efecto de la productividad de los RRHH sobre la innovación de producto $\left(0.727^{* *}\right)$ y el efecto de ésta sobre el desempeño exportador $\left(0.681^{*}\right)$ sí lo son, podemos afirmar que el análisis empírico sugiere, la existencia de una mediación total de la innovación de producto, en la relación entre la productividad de los RRHH y el desempeño exportador (Baron \& Kenny, 1986).

A continuación en la Tabla 3, podemos apreciar las correlaciones existentes entre todos los constructos del modelo completo. La Tabla 3 presenta la matriz de correlaciones de los constructos del modelo. 


\begin{tabular}{|r|l|r|r|r|r|r|r|r|}
\multicolumn{1}{l|}{} & Nombre del constructo & $\mathbf{1}$ & $\mathbf{2}$ & $\mathbf{3}$ & $\mathbf{4}$ & $\mathbf{5}$ & $\mathbf{6}$ & $\mathbf{7}$ \\
\hline $\mathbf{1}$ & Productividad de los RRHH & 1 & & & & & & \\
\hline $\mathbf{2}$ & Innovación de producto & $.728^{* *}$ & 1 & & & & \\
\hline $\mathbf{3}$ & Desempeño exportador & $.603^{* *}$ & $.796^{* *}$ & 1 & & & & \\
\hline $\mathbf{4}$ & Nivel tecnológico & $.016^{\text {n.s. }}$ & $.001^{\text {n.s. }}$ & $-.037^{\text {n.s. }}$ & 1 & & & \\
\hline $\mathbf{5}$ & Subsector & $-.054^{\text {n.s. }}$ & $-.037^{\text {n.s. }}$ & $-.084^{\text {n.s. }}$ & $-.0385^{* *}$ & & \\
\hline $\mathbf{6}$ & Edad & $-.039^{\text {n.s. }}$ & .025 & $0.120^{* *}$ & $0.067^{* *}$ & $-.054^{\text {n.s. }}$ & 1 & \\
\hline $\mathbf{7}$ & Tamaño & $0.043^{\text {n.s. }}$ & $0.196^{* *}$ & $0.360^{* *}$ & $0.014^{\text {n.s. }}$ & $-0.014^{\text {n.s. }}$ & $0.239^{* *}$ & 1 \\
\hline
\end{tabular}

$* \mathrm{p}<.05 ;{ }^{* *} \mathrm{p}<.01 ; * * * \mathrm{p}<.001 ;$ n.s.: No significativo.

Tabla 3. Correlación entre los constructos del modelo

\section{Conclusiones}

En este trabajo se ha examinado la relación entre la productividad laboral y el desempeño exportador de la PYME, proponiendo como variable mediadora la capacidad de innovación de producto. Ésta era nuestra tesis central. Para ello, se ha tomado la teoría de recursos y capacidades, como marco teórico, el cual se presenta especialmente valioso, dado su reconocido potencial para explicar la creación de ventajas competitivas sostenibles de las empresas (Volberda, 1996). El análisis empírico da apoyo a nuestra tesis central. La productividad de los recursos RRHH facilita el desarrollo de innovaciones de producto para incrementar el desempeño exportador. En este sentido, la innovación de producto es un mecanismo intermedio a través del cual, la mayor productividad de los RRHH, puede convertirse en un mayor desempeño exportador de la PYME manufacturera española. Este trabajo hace una gran contribución a la literatura sobre Dirección de Empresas en general, y Dirección Internacional y de la Innovación en particular, ya que explica conceptualmente y demuestra empíricamente, que la innovación de producto es un mecanismo intermedio clave, a través del cual, la mayor productividad de los RRHH, se convierte en un mayor desempeño exportador de la PYME manufacturera española.

Esta investigación tiene dos limitaciones principales:

- Los resultados aquí obtenidos sólo son generalizables a PYMES manufactureras españolas, innovadoras y que exportan con regularidad. La investigación futura podría explorar, si los resultados aquí obtenidos se sostienen en muestras de empresas de productos y/o servicios de otros países;

- este estudio sólo se centra en el rol de la innovación de producto, en la relación entre la productividad de los RRHH y el desempeño exportador; 
- también sería interesante estudiar el papel mediador de capacidades organizativas basadas en otros tipos de innovaciones (p.e., la innovación en procesos, organizativa o en marketing). El rol de la cultura organizativa (Barney, 1986) o/y los procesos de creación de conocimiento (Nonaka, 1994), en las relaciones entre productividad de los RRHH, innovación de producto y desempeño exportador. También parece una interesante oportunidad de investigación futura.

Esta investigación también proporciona lecciones a los directivos al sugerir a los empresarios de PYMES, que incrementos en la productividad de los RRHH, puede llevar a que dichos impactos sean capturados por la innovación, dando lugar a la generación de nuevos productos, que conllevará shocks de demanda, y en consecuencia de un mayor incremento de la exportación de sus empresas. Por otro lado, pretende concienciar a las políticas públicas, en la necesidad de sus ayudas a la financiación de la $\mathrm{I}+\mathrm{D}+\mathrm{i}$ de la PYMES, ya que esto aportaría mayor productividad de RRHH, lo que impactaría en el desarrollo de nuevos productos y por tanto, a mejores resultados empresariales y a una mayor internacionalización de las mismas.

\section{Referencias}

Andrés, A. (2011). El 80 por ciento de las empresas nuevas quiebran el primer año. La Razón, (19 de septiembre).

Baldauf, A., Cravens, D., \& Wagner, U. (2000). Examining determinants of export performance in small open economies. Journal of World Business, 35(1), 61-79. http://dx.doi.org/10.1016/S10909516(99)00034-6

Barney, J.B. (1986). Organizational culture: Can it be a source of sustained competitive advantage?. Academy of Management Review, 11(3), 656-665. http://dx.doi.org/10.5465/AMR.1986.4306261

Barney, J. (1991). Firm resources and sustained competitive advantage. Journal of Management, 17(1), 99-120. http://dx.doi.org/10.1177/014920639101700108

Baron, R., \& Kenny, D. (1986).The moderator-mediator variable distinction in social psychological research: Conceptual, strategic, and statistical considerations. Journal of Personality and Social Psychology, 51(6), 1173-1182. http://dx.doi.org/10.1037/0022-3514.51.6.1173

Basile, R. (2001). Export behaviour of Italian manufacturing firms over the nineties: The role of innovation. Research Policy, 30(8), 1185-1201. http://dx.doi.org/10.1016/S0048-7333(00)00141-4 
Becker, S.O., \& Egger, P.H. (2013). Endogenous product versus process innovation and a firm's propensity to export. Empirical Economics, 44(1), 329-354. http://dx.doi.org/10.1007/s00181-009-0322-6

Benítez, J., \& Ray, G. (2012). Introducing IT-enabled business flexibility and IT integration in the acquirer's M\&A performance equation. Proceedings of the 33rd International Conference on Information Systems, Orlando: Florida, USA: 1-21.

Benítez, J., \& Walczuch, R. (2012). Information technology, the organizational capability of proactive corporate environmental strategy and firm performance: A resource-based analysis. European Journal of Information Systems, 21(6), 664-679. http://dx.doi.org/10.1057/ejis.2012.14

Benítez, J., Lloréns, F.J., \& Férnandez, V. (2015). IT impact on talent management and operational environmental sustainability. Information Technology and Management, 16(3), 207-220. http://dx.doi.org/10.1007/s10799-015-0226-4

Bernard, A., \& Jensen, B. (2004). Why Some Firms Export. Review of Economics and Statistics, 86(2), 561-569. http://dx.doi.org/10.1162/003465304323031111

Boscherini, F., \& Yoguel, G. (1996). La capacidad innovativa y el fortalecimiento de la competitividad de las firmas: El caso de las PYMEs exportadoras argentinas. Buenos Aires: CEPAL (71).

Braojos, J., Benítez, J., \& Lloréns, J. (2015a). How do small firms learn to develop a social media competence?. International Journal of Information Management, 35(4), 443-458. http://dx.doi.org/10.1016/j.jijnfomgt.2015.04.003

Braojos, J., Benítez, J., \& Lloréns, J. (2015b). Impact of IT infrastructure on customer service performance: The role of micro-IT capabilities and online customer engagement. Proceedings of the 19th Pacific Asia Conference on Information Systems, Singapore: 1-17.

Cenfetelli, R., \& Bassellier, G. (2009).Interpretation of Formative Measurement in Information Systems Research. MIS Quarterly, 33(4), 689-707.

Chen, I., Paulraj, A., \& Lado, A. (2004). Strategic purchasing, supply management, and firm performance. Journal of Operations Management, 22(5), 505-523. http://dx.doi.org/10.1016/j.jom.2004.06.002

Chin, W., Marcolin, B., \& Newsted, P. (2003). A Partial Least Squares Latent Variable Modeling Approach for Measuring Interaction Effects: Results from a Monte Carlo Simulation Study and an Electronic-Mail Emotion/Adoption Study. Information Systems Research, 14(2), 189-217. http://dx.doi.org/10.1287/isre.14.2.189.16018 
CNAE-2009. Real Decreto 475/2007, de 13 de abril, por el que se aprueba la Clasificación Nacional de Actividades Económicas 2009. BOE, 102: 207-223. Disponible online en: http://www.ine.es/jaxi/menu.do? type $=$ pcaxis $\&$ path $=/ \mathrm{t} 40 /$ clasrev\&file $=$ inebase

Cook, T.D., Campbell, D.T., \& Day, A. (1979). Quasi-experimentation: Design \& analysis issues for field settings (Vol. 351). Boston: Houghton Mifflin.

Czinkota, M.R., \& Johnston, W.J. (1983). Exporting: Does sales volume make a difference?. Journal of International Business Studies, 14, 147-153. http://dx.doi.org/10.1057/palgrave.jibs.8490513

Damanpour, F. (1991). Organizational innovation: A meta-analysis of effects of determinants and moderators. Academy of Management Journal, 34, 555-590. http://dx.doi.org/10.2307/256406

Damanpour, F., \& Aravind, D. (2006). Product and process innovations: A review of organizational and environmental determinants. En J. Hage \& M. Meeus (eds.), Innovation, Science, and Industrial Change: A Research Handbook (pp. 38-66). Oxford University Press.

Eaton, J., Kortum, S., \& Kramarz, F. (2011). An anatomy of international trade: Evidence from French firms. Econometrica, 79(5), 1453-1498. http://dx.doi.org/10.3982/ECTA8318

Eisenhardt, K., \& Martin, A. (2000). Dynamic capabilities: What are they?. Strategic Management Journal, 21(10-11), 1105-1121. http://dx.doi.org/10.1002/1097-0266(200010/11)21:10/11<1105::AID-SMJ133>3.0.CO;2$\mathrm{E}$

Eusebio, R., Llonch A., \& Pilar, L. (2006). Measures of marketing performance: A comparative study from Spain. International Journal of Contemporary Hospitality Management, 18(2), 145-155. http://dx.doi.org/10.1108/09596110610646691

Fornell, C. (1982). A Second Generation of Multivariate Analysis: An Overview. In C. Fornell (ed.), $A$ Second Generation of Multivariate Analysis (pp. 1-21). Nueva York: Praeger Publishers.

García, F., \& Avella, L. (2008). La influencia de la exportación sobre los resultados empresariales: Análisis de las PYMES manufactureras españolas en el periodo 1991-2002. Revista Europea de Dirección y Economía de la Empresa, 17(2), 85-104.

Garcia, R., \& Calantone, R. (2002). A critical look at technological innovation typology and innovativeness terminology: A literature review. Journal of Pproduct Innovation Management, 19(2), 110-132. http://dx.doi.org/10.1016/S0737-6782(01)00132-1

Hair, J.F., Ringle, C.M., \& Sarstedt, M. (2013). Editorial-partial least squares structural equation modeling: Rigorous applications, better results and higher acceptance. Long Range Planning, 46(1-2), 1-12. http://dx.doi.org/10.1016/j.lrp.2013.01.001 
Henderson, R., \& Cockburn, I. (1994). Measuring Competence - Exploring Firm Effects in Pharmaceutical Research. Strategic Management Journal, 15: 63-84.http://dx.doi.org/10.1002/smj.4250150906

ICEX (2014). España, Exportación e Inversiones. Ministerio de Economía y Competitividad. Gobierno de España. Disponible online en: http://www.icex.es/icex/es/index.html

Jarvis, C.B., Mackenzie, S.B., \& Podsakoff, P.M. (2003). A critical review of construct indicators and measurement model misspecification in marketing and consumer research. Journal of Consumer Research, 30(2), 199-218. http://dx.doi.org/10.1086/376806

Jaumandreu, J. (2009). What explains the evolution of productivity and competitiveness? The innovation link. Working Paper No 804, IESE Business School, University of Navarra. http://dx.doi.org/10.2139/ssrn.1482990

Katsikeas, C.S., Leonidou, L.C., \& Morgan, N.A. (2000). Firm-level export performance assessment: review, evaluation, and development. Journal of the Academy of Marketing Science, 28(4), 493-511. http://dx.doi.org/10.1177/0092070300284003

Kemppilä, S., \& Lönnqvist, A. (2003). Subjective productivity measurement. The Journal of American Academy of Business, 2(2), 531-537.

Kugler, M., \& Verhoogen, E. (2008). The quality-complementarity hypothesis: Theory and evidence from Colombia (No. w14418). National Bureau of Economic Research. http://dx.doi.org/10.3386/w14418

Lagarda, A.M., \& Urquidy, M.R. (2007). Capital humano y productividad en microempresas. Investigación Económica, 66(260), 81-115.

Leonidou, L. (2004). An analysis of the barriers hindering small business export development. Journal of Small Business Management, 42(3), 279-302. http://dx.doi.org/10.1111/j.1540-627X.2004.00112.x

Leonidou, L.C., Katsikeas, C.S., \& Samiee, S. (2002). Marketing strategy determinants of export performance: A meta-analysis. Journal of Business Research, 55(1), 51-67. http://dx.doi.org/10.1016/S01482963(00)00133-8

Luthans, F., \& Youssef, C.M. (2004). Human, Social, and Now Positive Psychological Capital Management: Investing in People for Competitive Advantage. Organizational Dynamics, 33(2), 143-160. http://dx.doi.org/10.1016/j.orgdyn.2004.01.003

Martín, M., \& Rodríguez, C. (2009). Una aproximación a las características de las empresas exportadoras españolas. Boletín Económico. Banco de España, (5), 43-55. 
Moori, V., Milesi, D., Robert, V., \& Yoguel, G. (2007). Desarrollo de ventajas competitivas: PYMES exportadoras exitosas en Argentina, Chile y Colombia. Revista de la CEPAL, 92, 25-43.

Molina-Domene, M., \& Pietrobelli, C. (2012). Drivers of technological capabilities in developing countries: An econometric analysis of Argentina, Brazil and Chile. Structural Change and Economic Dynamics, 23(4), 504-515. http://dx.doi.org/10.1016/j.strueco.2011.11.003

Munuera, J., \& Rodríguez, A. (2007). Estrategias de marketing. Un enfoque basado en el proceso de dirección. Madrid: ESIC Editorial.

Nonaka, I. (1994). A dynamic theory of organizational knowledge creation. Organization Science, 5(1), 14-37. http://dx.doi.org/10.1287/orsc.5.1.14

Núñez, P., Grande, F., \& Lorenzo, D. (2015). The effects of coaching in employees and organizational performance: The Spanish case. Intangible Capital, 11(2), 166-189.

OCDE (2005). The measurement of scientific and technological activities. Oslo Manual. Guidelines for collecting and interpreting innovation data. OCDE EUROSTAT, París. $3^{\text {a }}$ ed.

OCDE (2015). Taxation of SMEs in OECD and G20 Countries, OECD Tax Policy, Studies, No. 23, OCDE, Publishing, Paris.

PITEC (2013). Panel de Innovación Tecnológica. Disponible online en: http://icono.fecyt.es/PITEC.aspx (Fecha último acceso: 29 de septiembre, 2015)

Prahalad, C.K., \& Hamel, G. (1990). The Core Competence of the Corporation. Harvard Business Review, 68(3), 79-91.

Preacher, K., \& Hayes, A. (2008). Asymptotic and resampling strategies for assessing and comparing indirect effects in multiple mediator models. Behavior Research Methods, 40(3), 879-891. http://dx.doi.org/10.3758/BRM.40.3.879

Quijano, S. (2006). Dirección de Recursos Humanos y Consultoría en las Oganizaciones (vol.74, pp.432). Icaria: Barcelona, España.

Rialp, A., \& Rialp, J. (2001). Conceptual frameworks on SMEs' internationalization: Past, present and future trends of research. In C.N. Axinn, \& P. Matthyssens (Eds.), Reassesing the Internationalization of the Firm (pp. 49-78). Amsterdam: Emerald Group Publishing Limited.

Ringle, C.M., Wende, S., \& Will, S. (2005). SmartPLS 2.0 (M3) Beta, Hamburg. 
Ruizalba, J., Vallespín, M., Martín, V., \& Rodríguez, M. (2015). The moderating role of gender on entrepreneurial intentions: A TPB perspective. Intangible Capital, 11(1), 92-117. http://dx.doi.org/10.3926/ic.557

Saari, L.M., \& Judge, T.A. (2004). Employee attitudes and job satisfaction. Human Resource Management, 43(4), 395-407. http://dx.doi.org/10.1002/hrm.20032

Sánchez, F., Hernández, G., \& Prada, M. (2004). Crecimiento de las exportaciones y sus efectos sobre el empleo, la desigualdad y la pobreza en Colombia (Vol. 3). CEDE, Centro de Estudios sobre Desarrollo Económico.

Teece, D. (2007). Explicating Dynamic Capabilities: The Nature and Microfoundations of (Sustainable) Enterprise Performance. Strategic Management Journal, 28(13), 1319-1350. http://dx.doi.org/10.1002/smj.640

Teece, D., \& Pisano, G. (1994).The Dynamic Capabilities of Firms: An Introduction. Industrial and Corporate Change, 3(3), 537-556. http://dx.doi.org/10.1093/icc/3.3.537-a

Teece, D., Pisano, G., \& Schuen, A. (1997). Dynamic capabilities and Sstrategic Management. Strategic Management Journal, 18(7), 509-533. http://dx.doi.org/10.1002/(SICI)1097-0266(199708)18:7<509::AIDSMJ882>3.0.CO;2-Z

Visintin, S., Di Meglio, G., Rubalcaba, L., \& Cuadrado, J.R. (2008). Competitividad y comercio internacional de servicios en España. Papeles de Economía Española, (116), 65-78.

Volberda, H. (1996). Toward the flexible form: How to remain vital in hypercompetitive environments. Organization Studies Science, 7(4), 359-374. http://dx.doi.org/10.1287/orsc.7.4.359

Wang, Y., Chen, Y., \& Benitez-Amado, J. (2015). How information technology influences environmental performance: empirical evidence from China. International Journal of Information Management, 35(2), 160-170. http://dx.doi.org/10.1016/j.ijinfomgt.2014.11.005

Zhao, X., Lynch, J., \& Chen, Q. (2010). Reconsidering Baron and Kenny: Myths and Truths about Mediation Analysis. Journal of Consumer Research, 37(2), 197-206. http://dx.doi.org/10.1086/651257 


\section{Anexo A: Nombre del constructo, definición de la medida y fuente del dato}

\begin{tabular}{|c|c|c|}
\hline $\begin{array}{l}\text { Nombre del } \\
\text { constructo }\end{array}$ & Definiciónde la medida & $\begin{array}{l}\text { Fuente } \\
\text { del dato }\end{array}$ \\
\hline $\begin{array}{l}\text { Productividad } \\
\text { de los RRHH }\end{array}$ & $\begin{array}{l}\text { Variable observada tomada como la cifra de negocio total del año } 2011 \text { (nombre } \\
\text { de la variable en PITEC: "cifra", divida por el número de empleado del mismo } \\
\text { año (nombre de la variable en PITEC: "tamaño"). }\end{array}$ & PITEC \\
\hline $\begin{array}{l}\text { Innovación de } \\
\text { producto }\end{array}$ & $\begin{array}{l}\text { Este constructo ha sido medido mediante dos variables observadas, una de ellas } \\
\text { (nombre de la variable en PITEC: "newemp") mide el término innovación en } \\
\text { relación con el desarrollo de nuevos productos desde una óptica interna, es decir, } \\
\text { de las novedades o cambios que introduce la empresa en sus productos, y la } \\
\text { segunda variable observada (nombre de la variable en PITEC: "newmer") mide la } \\
\text { innovación de productos desde una perspectiva externa o de mercado, esto es, } \\
\text { como la introducción de productos que suponen alguna novedad para el } \\
\text { mercado. Ambas medidas han sido consideradas como porcentaje de la cifra total } \\
\text { de negocio de la PYME para el año } 2013 \text {. }\end{array}$ & PITEC \\
\hline $\begin{array}{l}\text { Desempeño } \\
\text { exportador }\end{array}$ & $\begin{array}{l}\text { Medido por dos variables observadas: la primera variable observada es la } \\
\text { intensidad exportadora (nombre de las variables en PITEC: "exportn" e } \\
\text { "intracom"), medida por el porcentaje de ventas en el mercado foráneo en } \\
\text { relación a la cifra total de negocio de la PYME, y por una segunda variable } \\
\text { observada, la experiencia exportadora, medida por el número de años que la } \\
\text { empresa ha exportado desde su creación (nombre de la variable en PITEC que } \\
\text { informa del año de creación de la PYME: "aniocrea") }\end{array}$ & PITEC \\
\hline $\begin{array}{l}\text { Nivel } \\
\text { tecnológico }\end{array}$ & $\begin{array}{l}\text { Variable observada creada según la clasificación sectorial definida por CNAE- } \\
\text { 2009: a partir de la variable denominada en PITEC como "actin" que identifica el } \\
\text { subsector de actividad. Se ha codificado esta variable como: NIVEL ALTA } \\
\text { TECNOLOGÍA=1; NIVEL MEDIA-ALTA TECNOLOGÍA=2; NIVEL } \\
\text { MEDIA-BAJA TECNOLOGÍA=3; NIVEL BAJA TECNOLOGÍA=4 }\end{array}$ & PITEC \\
\hline Subsector & $\begin{array}{l}\text { Variable observada que mide el subsector de la actividad (nombre de la variable } \\
\text { en PITEC: "actin" }\end{array}$ & PITEC \\
\hline Edad & $\begin{array}{l}\text { Variable observada medida como el número de años de actividad de la empresa } \\
\text { (nombre de la variable en PITEC que indica el año de creación: "aniocrea") }\end{array}$ & PITEC \\
\hline Tamaño & $\begin{array}{l}\text { Variable observada (nombre de la variable en PITEC: "tamano") medido a través } \\
\text { del número de la empleados de la PYME durante el año } 2013 .\end{array}$ & PITEC \\
\hline
\end{tabular}

Intangible Capital, 2016 (www.intangiblecapital.org)

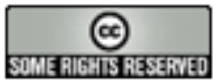

Article's contents are provided on an Attribution-Non Commercial 3.0 Creative commons license. Readers are allowed to copy, distribute and communicate article's contents, provided the author's and Intangible Capital's names are included. It must not be used for commercial purposes. To see the complete license contents, please visit http://creativecommons.org/licenses/by-nc/3.0/. 